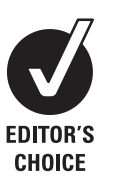

arc Epidemiology Unit,

Manchester Academic Health Sciences Centre, University of Manchester, Manchester, UK 2Department of Biostatistics, Manchester Academic Health Sciences Centre, University of Manchester, Manchester, UK ${ }^{3}$ arc National Primary Care Centre, Keele University, Keele, Staffs, UK and Department of Rheumatology, Haywood Hospital, Stoke-on-Trent, UK

Correspondence to Professor D P M Symmons, arc Epidemiology Unit, Stopford Building, The University of Manchester, Manchester M13 9PT, UK; deborah.symmons@ manchester.ac.uk

Accepted 17 September 2009 Published Online First 8 November 2009

\title{
Beneficial effects of a 3-week course of intramuscular glucocorticoid injections in patients with very early inflammatory polyarthritis: results of the STIVEA trial
}

\author{
S M M Verstappen, ${ }^{1}$ M J McCoy, ${ }^{1}$ C Roberts, ${ }^{2}$ N E Dale, ${ }^{1}$ A B Hassell, ${ }^{3}$ \\ D P M Symmons, ${ }^{1}$ the STIVEA investigators
}

\begin{abstract}
Objective To evaluate whether treating patients with very early inflammatory polyarthritis (IP) with a 3-week course of intramuscular (IM) methylprednisolone acetate may postpone the need for disease-modifying antirheumatic drugs (DMARDs) and prevent IP from evolving into rheumatoid arthritis (RA).
\end{abstract}

Methods Patients with very early IP (4-10 weeks' duration) were randomised to receive three injections of either $80 \mathrm{mg}$ IM methylprednisolone acetate or placebo, given at weekly intervals. Assessments were monthly until 6 months after the first injection, and then concluded at 12 months. The primary outcome was the need to start DMARDs by the 6-month assessment. Secondary outcomes included disease activity and final clinical diagnosis by the rheumatologist at 12 months.

Results Patients in the placebo group (76\%) were more likely to need DMARDs during the first 6 months of the trial than patients in the glucocorticoid group (61\%) (adjusted $\mathrm{OR}=2.11,95 \% \mathrm{Cl} 1.16$ to $3.85, \mathrm{p}=0.015$ ). Disease activity did not differ between the two groups at 12 months, probably because many patients in the placebo group started DMARDs early in the study. After 12 months, the arthritis had resolved without the need for DMARDs in $9.9 \%(11 / 111)$ of the patients in the placebo group and in 19.8\% (22/111) in the glucocorticoid-treated group (adjusted $\mathrm{OR}=0.42,95 \% \mathrm{Cl} 0.18$ to 0.99 , $\mathrm{p}=0.048$ ).

Conclusion Treatment of patients with very early IP with IM methylprednisolone acetate appears to postpone the prescription of DMARDs and prevent one in 10 patients from progressing into RA.

The body normally responds either locally or systemically to an inflammatory stimulus by producing proinflammatory cytokines. Systemically, the release of proinflammatory cytokines results in stimulation of the hypothalamicpituitary-adrenal axis and release of corticosteroids. This should then curtail the inflammation. Inflammatory polyarthritis (IP) is an example of a systemic inflammatory stimulus, but not all people who develop IP go into remission. Evidence from the Norfolk Arthritis Register suggests that at least half of those who develop IP lasting at least 4 weeks go on to develop chronic rheumatoid arthritis (RA). ${ }^{1}$ One possible explanation for the persistence of IP may be a defect in the patient's systemic anti-inflammatory response. Reduced corticosteroid or ACTH levels have been found in patients with RA, whereas one would expect elevated levels due to inflammation. ${ }^{2-4}$

Oral glucocorticoids are commonly prescribed to patients with RA because of their clinical efficacy. ${ }^{5-7}$ However, only a few randomised controlled trials have examined the effect of intramuscular (IM) administration of glucocorticoids in patients with inflammatory arthritis. In three randomised controlled trials, IM glucocorticoids were given at the same time as starting disease-modifying antirheumatic drugs (DMARDs). ${ }^{8-10}$ In one study no apparent benefit was shown from the glucocorticoid injections. ${ }^{10}$ In two other studies, IM glucocorticoid injections had a significant short-term benefit when compared with either oral glucocorticoids or placebo. $^{89}$ These results were confirmed in another study, comparing monthly IM glucocorticoid injections with placebo in patients with established RA, in which a significant reduction in disease activity up to 6 months was found. ${ }^{11}$ In the latter study, however, the number of reported adverse events outweighed the observed clinical benefit. Although these studies show that the use of IM glucocorticoids is in general very beneficial, disease duration in these studies was more than 3 months and most patients had already developed RA.

In recent years it has become clear that the "window of opportunity" to fundamentally alter the course of RA is very narrow and that treatment should start as early as possible after symptom onset. ${ }^{12-14}$ A previously published non-randomised study, exploring the effect of a single dose of glucocorticoids in patients with very early arthritis, showed that patients who went into remission had a median symptom duration of 10 weeks compared with a median duration of 20 weeks in those whose arthritis persisted. ${ }^{15}$ Thus we hypothesised that administration of IM glucocorticoid injections in very early IP may lead to resolution in an important proportion of cases.

\section{PATIENTS AND METHODS \\ Study design and patients}

This randomised, double-blind, placebo-controlled, multicentre trial examined the effect of IM glucocorticoids in patients with very early IP. General practitioners in the region of 23 participating hospitals were asked to refer patients aged 18 or older who had IP with symptom duration of 4-10 weeks to the rheumatology outpatient clinic. Patients had to have tenderness and soft tissue 
swelling of two or more joints, at least one of which had to be a wrist, metacarpophalangeal or proximal interphalangeal joint. Rheumatologists aimed to see these patients within 7 days of referral. Exclusion criteria included sepsis, proven or suspected crystal arthritis, known arthritis diagnosis (eg, systemic lupus erythematosus, osteoarthritis), previous history of IP, serious comorbidities, diabetes mellitus, epilepsy, bleeding disorder, glaucoma, peptic ulceration, pregnancy, treatment with oral glucocorticoids or local glucocorticoids within the past 6 months, known allergy to glucocorticoids or extra-articular features. The North West Research Ethics Committee UK approved the trial and all patients gave written informed consent.

\section{Treatment and visit assessments}

One week after screening, patients were randomised into the glucocorticoid or the placebo arm via telephone by a coordinating centre in Manchester using a computerised program which employs "minimisation" techniques. ${ }^{16}$ Stratification for the following variables occurred: age $(<45,45-64, \geqslant 65)$, gender, centre, symptom duration ( $<8$ weeks, $\geqslant 8$ weeks) and "delayed entrance" (seen $\leqslant 7$ days from referral, $>7$ days). At this baseline visit, patients received the first of three injectionsthat is, either $80 \mathrm{mg}$ IM methylprednisolone acetate $(2 \mathrm{ml}$ of $40 \mathrm{mg} / \mathrm{ml}$ methylprednisolone acetate) (glucocorticoid group) or $2 \mathrm{ml}$ normal saline (placebo group) deep into the gluteal muscle. Two further injections of either $80 \mathrm{mg}$ methylprednisolone acetate or saline were given at weekly intervals. To maintain a somewhat higher and continuous level of glucocorticoids we chose to give $80 \mathrm{mg}$ weekly instead of one injection of $120 \mathrm{mg}$ expecting a serum peak of $43.5 \mathrm{ng} / \mathrm{ml}$ at $6-8 \mathrm{~h}, 8 \mathrm{ng} /$ $\mathrm{ml}$ at day 7 and undetectable at day $18 .{ }^{17}$ A nurse, who was not involved in conducting the assessments, drew up and administered the methylprednisolone acetate or saline. After completing the course of injections, patients were seen in the hospital by a research nurse at 1-month intervals until 6 months after the baseline visit and then at 12 months. The patient, rheumatologist and research nurse were blinded to treatment allocation.

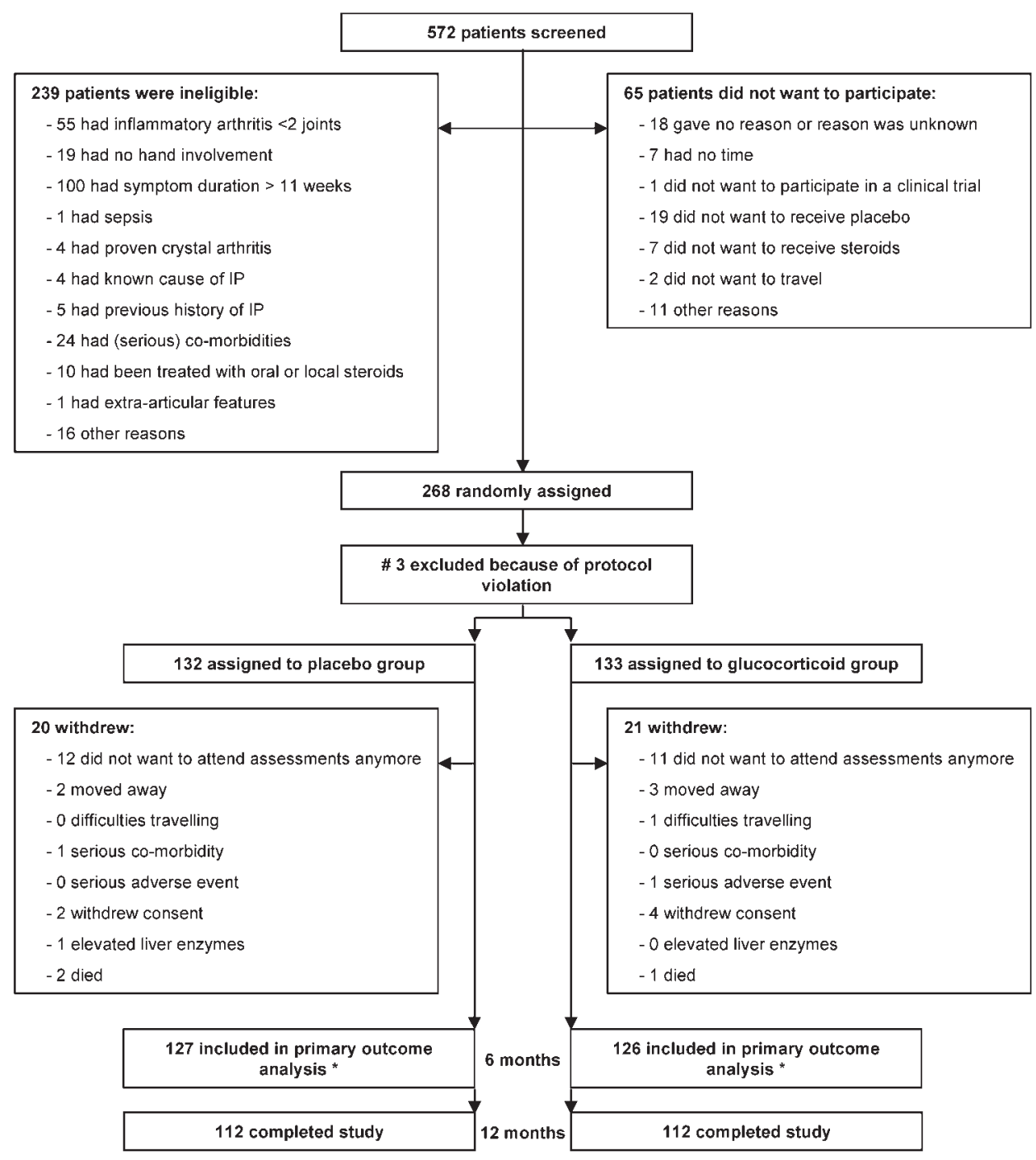

Figure 1 Trial profile. \#One patient was randomised in advance. One patient did not receive any medication owing to a mix up with the pharmacist, so the rheumatologist withdrew this patient from the study. The third patient signed the consent form, but no clinical data were available for this patient and symptom duration on inclusion form was $>1$ year. ${ }^{*} 253$ patients were included in the primary outcome analyses. 230 had a follow-up duration of $>6$ months; 115 in the placebo group and 115 in the glucocorticoid group. The other 23 patients (12 patients in the placebo group and 11 patients in the glucocorticoid group) were lost to follow-up in the first 6 months because they did not want to attend for the assessments anymore, but data on disease-modifying antirheumatic drug use were obtained from their GPs. IP, inflammatory polyarthritis. 
Table 1 Baseline characteristics

\begin{tabular}{|c|c|c|c|c|}
\hline \multirow[b]{2}{*}{ Characteristics } & \multicolumn{2}{|c|}{ Placebo group } & \multicolumn{2}{|c|}{ Glucocorticoid group } \\
\hline & N & Value & $\mathbf{N}$ & Value \\
\hline Gender, female, n (\%) & 132 & $91(69)$ & 133 & $91(68)$ \\
\hline Age (years), mean (SD) & 132 & $56(15)$ & 133 & $55(15)$ \\
\hline Symptom duration (weeks), mean (SD) & 128 & $8.0(2.3)$ & 132 & $7.7(2.6)$ \\
\hline $\operatorname{ESR}(\mathrm{mm} / \mathrm{h})$, median $\left(I \mathrm{Q}_{0.25-0.75}\right)$ & 129 & $30(14-48)$ & 130 & $27(15-47)$ \\
\hline Total number of swollen joints, ${ }^{*}$ median $\left(10_{0.25-0.75}\right)$ & 131 & $8(4-12)$ & 132 & $8(4.5-12)$ \\
\hline Total number of tender joints, ${ }^{*}$ median $\left(\mathrm{IO}_{0.25-0.75}\right)$ & 131 & $9(5-15)$ & 132 & $9(5-14)$ \\
\hline $\operatorname{DAS28}(3)$, mean $(S D)$ & 128 & $5.3(1.2)$ & 129 & $5.3(1.2)$ \\
\hline VAS pain $(\mathrm{mm})$, mean (SD) & 126 & $54(24)$ & 129 & $55(25)$ \\
\hline VAS fatigue (mm), mean (SD) & 126 & $53(28)$ & 129 & $53(26)$ \\
\hline VAS physician (mm), mean (SD) & 132 & $49(19)$ & 131 & $47(20)$ \\
\hline Early morning stiffness (min), median $\left(\mathrm{IO}_{0.25-0.75}\right)$ & 90 & $60(15-120)$ & 84 & $60(30-120)$ \\
\hline Radiological damage, yes, n (\%) & 102 & $12(12)$ & 103 & $12(11.7)$ \\
\hline Rheumatoid factor, positive, n (\%) & 127 & $44(35)$ & 130 & $40(31)$ \\
\hline $\mathrm{HAO}$ score, mean $(\mathrm{SD})$ & 126 & $1.21(0.63)$ & 129 & $1.17(0.79)$ \\
\hline EQ-5D utility score, median $\left(\mid \mathrm{Q}_{0.25-0.75}\right)$ & 121 & $0.59(0.12-0.69)$ & 127 & $0.62(0.19-0.69)$ \\
\hline SF-36 PCS score, mean (SD) & 123 & $39(6)$ & 122 & $39(7)$ \\
\hline SF-36 MCS score, mean (SD) & 123 & $44(7)$ & 122 & $45(7)$ \\
\hline
\end{tabular}

$\mathrm{N}$, number of patients with data.
${ }^{*}$ Number of swollen or tender joints (0-28 maximum).
DAS28(3), three-component 28 joint Disease Activity Score (maximum score $=10$ ); EQ-5D, Euro0ol-5D dimension utility score
(range 1 to $-0.59=$ worst health); ESR, erythrocyte sedimentation rate; HAQ, Health Assessment Score (range $0-3=$ worst
functional ability); MCS, SF-36 mental component summary score; PCS, SF-36 physical component summary score (higher scores
indicate better health); VAS, visual analogue scale (range $0-100 \mathrm{~mm}$, worst score).

At baseline and all subsequent visits, the three-component 28 -joint disease activity score (DAS28(3)) $)^{18}$ was calculated based on a 28 swollen joint count, a 28 tender joint count and the erythrocyte sedimentation rate. In addition, duration of morning stiffness and patients' assessment on visual analogue scales (VAS) for pain and for fatigue were recorded. The baseline, 6- and 12-month visits also included a health utility score (EuroQol-5D) ${ }^{19}$ and the SF- $36^{20}$ physical component score and mental component score filled in by the patient. Disease activity according to the rheumatologist (VAS physician), rheumatoid factor (positive if $>40 \mathrm{IU} / \mathrm{l}$ ), functional disability (British version of the Health Assessment Questionnaire ${ }^{21}$ ) and $x$-ray reports of hands and feet were obtained at baseline and at 1 year.

Once patients satisfied three or more of the following criteria, as defined in the protocol, they were referred to the rheumatologist with a view to starting DMARDs: three or more swollen joints, six or more tender joints, at least 45 min early morning stiffness or erythrocyte sedimentation rate $\geqslant 28 \mathrm{~mm} / \mathrm{h}$. These values were chosen because they are common entry criteria for DMARD clinical trials. Once patients were referred for DMARDs, they no longer attended the hospital for monthly assessments but were evaluated at 6 and 12 months by the research nurse and the rheumatologist.

Administration of intra-articular glucocorticoid injections and/or additional IM glucocorticoid injections was allowed at any time, if clinically indicated, after completion of the injection course. Data on injections were obtained retrospectively from the medical records. Patients who were using non-steroidal antiinflammatory drugs (NSAIDs) at study start were asked to continue using them until at least 1 week after the treatment injections had been given. Thereafter, NSAIDs could be started or stopped at any time during follow-up.

\section{Primary outcome}

The primary outcome measure was the need to start DMARDs by the 6-months' assessment - that is, the referral of the patient to the rheumatologist by the nurse to consider DMARDs (including oral glucocorticoids), according to the above mentioned criteria, or the actual start of DMARDs by 6 months. To allow for variation in the timing of the 6-month visit assessment, a time period up to seven months from the baseline visit was chosen in advance. All patients for whom follow-up data were available until at least the 6-month assessment were included in this analysis. In addition, patients who were lost to follow-up, either before or after the 6-months' assessment, because they did not want to attend the clinic anymore were also included in this analysis. The general practitioners of these patients were contacted to establish if they had started DMARDs at any time up to 12 months from the first injection.

\section{Secondary outcomes}

The secondary outcome measures included (a) the difference in disease activity measures, functional disability and utility measures between the two groups at 6 months and at 1 year; (b) a comparison between the two treatment groups of the number of patients with no joint damage at baseline who developed erosions at 1 year based on $x$-ray reports obtained from the department of radiology at each hospital; and (c) the final clinical diagnosis determined by the rheumatologist at 12 months categorised into: RA, resolved, undifferentiated IP, non-inflammatory disease and other inflammatory diseases.

\section{Sample size}

Based on observational data from the Norfolk Arthritis Register cohort, we estimated that $75 \%$ of the placebo-treated group would require DMARDs by 6 months after starting the course of injections. A total sample size of 262 would be needed to detect a fall in the need for DMARDs of $25 \%$ (which is equivalent to a failure rate of $56 \%$ in the treated group) with $80 \%$ power and $5 \%$ significance level and allowing for $15 \%$ loss to follow-up.

\section{Statistical analyses}

Analysis for the primary outcome was carried out according to intention-to-treat, subject to the availability of data. 


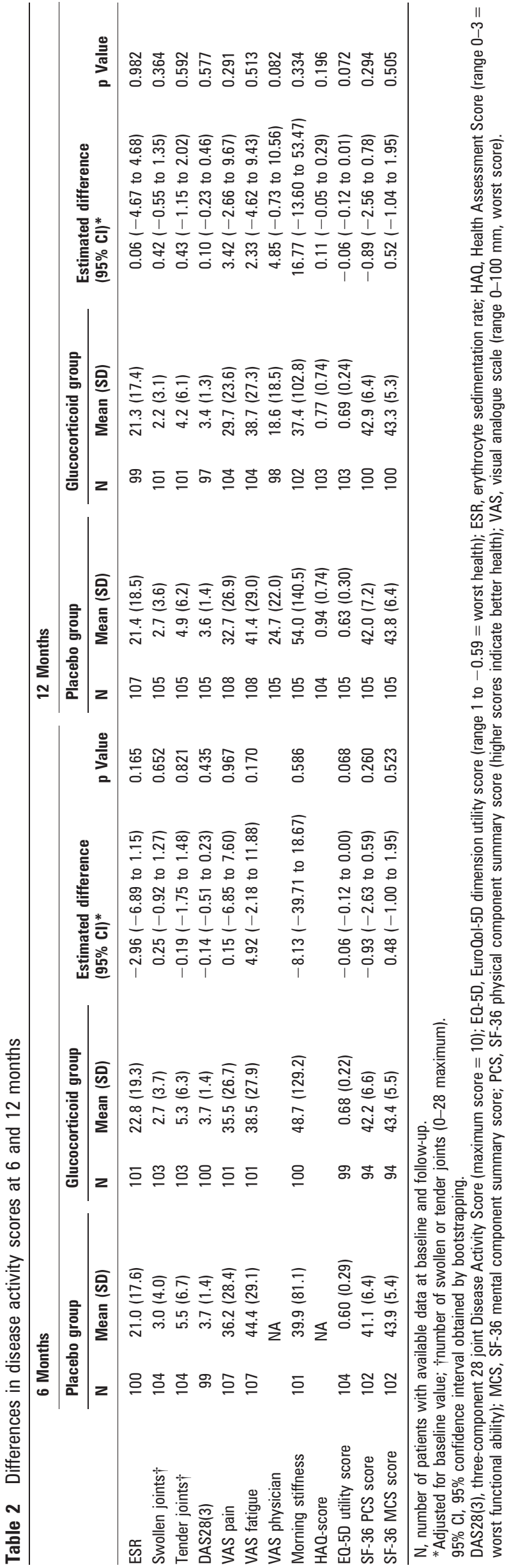

Table 3 Final diagnosis by a doctor at 1 year

\begin{tabular}{lll}
\hline Final diagnosis & $\begin{array}{l}\text { Placebo group } \\
(\mathbf{n = 1 1 1 )}\end{array}$ & $\begin{array}{l}\text { Glucocorticoid group } \\
(\mathbf{n}=\mathbf{1 1 1})\end{array}$ \\
\hline RA & $67(60.4)$ & $54(48.6)$ \\
Used DMARDs & 59 & 48 \\
No DMARDs & 8 & 6 \\
Undifferentiated IP & $19(17.1)$ & $22(19.8)$ \\
Used DMARDs & 12 & 9 \\
No DMARDs & 7 & 13 \\
Resolved/remission & $13(11.7)$ & $23(20.7)$ \\
Used DMARDs & 2 & 1 \\
No DMARDs & 11 & 22 \\
& & \\
Non-inflammatory disease & $6(5.4)$ & $9(8.1)$ \\
Used DMARDs & 1 & 1 \\
No DMARDs & 5 & 8 \\
Other inflammatory diseases & $6(5.4)$ & $3(2.7)$ \\
Used DMARDs & 5 & 1 \\
No DMARDs & 1 & 2 \\
\hline
\end{tabular}

Results are shown as number (\%) or number.

DMARDs, disease-modifying antirheumatic drugs; IP, inflammatory polyarthritis; RA, rheumatoid arthritis.

Additionally, two separate sensitivity analyses were performed for the primary outcome measure. First, all patients $(n=12)$ with no data on DMARDs were considered not to have received DMARDs. Second, these patients were considered to have received DMARDs. Logistic regression analyses were conducted to determine the odds ratio (OR) for the need to start DMARDs within 6 months, the clinical diagnosis of RA at 1 year and whether the arthritis had resolved at 1 year. ORs were adjusted for age, gender, symptom duration (calculated as the difference between the date of the first visit and date of onset of symptoms), VAS for pain, Health Assessment Questionnaire score, DAS28(3) and rheumatoid factor (RF) assessed at baseline. ${ }^{22}$ Disease activity differences, adjusted for baseline values, between the two groups were tested using linear regression. Confidence intervals were obtained by bootstrapping (1000 replications). For logistic and linear regression analyses, missing baseline data were imputed by age- and gender-associated scores. ${ }^{23} \chi^{2}$ Statistics were applied to test the difference in percentage of patients with new erosions at follow-up. KaplanMeier survival curves were used to estimate the median time

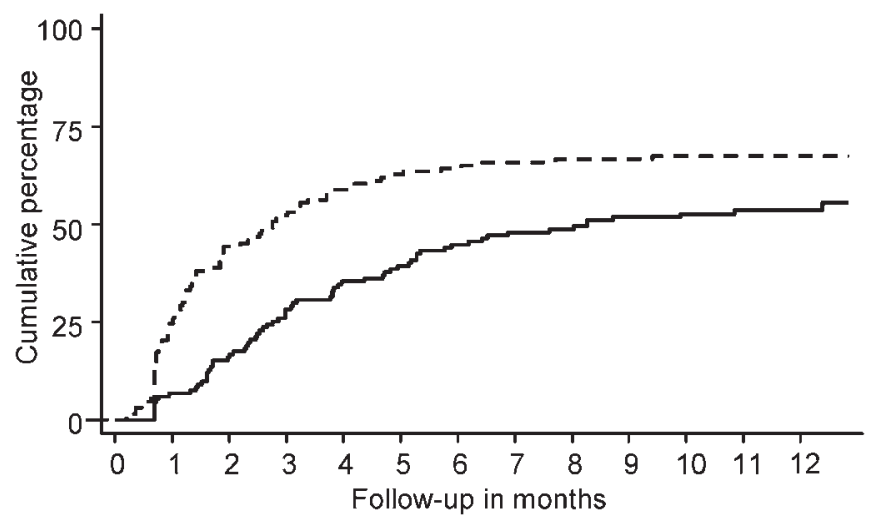

Figure 2 The cumulative percentage for starting disease-modifying antirheumatic drugs in the glucocorticoid-treated arm (solid line) and the placebo-treated arm (dashed line). 
Table 4 Adverse events (AEs)

\begin{tabular}{|c|c|c|c|c|}
\hline Description of event & $\begin{array}{l}\text { Outcome at time of } \\
\text { reporting AEs }\end{array}$ & $\begin{array}{l}\text { Related to } \\
\text { treatment }\end{array}$ & $\begin{array}{l}\text { Time* } \\
\text { (months) }\end{array}$ & DMARDs $\uparrow$ \\
\hline \multicolumn{5}{|l|}{ Placebo group } \\
\hline \multicolumn{5}{|l|}{ Adverse reactions } \\
\hline Admitted to hospital with severe abdominal pain & Resolved completely & Probably & 1.4 & No \\
\hline Headache & Resolved completely & Probably & 0.07 & No \\
\hline Nausea & Resolved completely & Probably & 0.07 & No \\
\hline \multicolumn{5}{|l|}{ Adverse events } \\
\hline Developed lung cancer & Patient died & Probably not & 3.2 & No \\
\hline Developed breast cancer & Resolved partially & Probably not & 6.7 & Yes \\
\hline Anaphylactic shock due to Zantac & Resolved completely & No & 4.9 & No \\
\hline Metastatic adenocarcinoma & Not resolved & No & 10.0 & Yes \\
\hline Pancreatitis & Resolved completely & No & 1.8 & No \\
\hline Pleural effusion & Resolved partially & No & 6.8 & Yes \\
\hline Carcinoma of sigmoid colon & Not known & No & 7.8 & Yes \\
\hline Carcinoma of prostate & Resolved partially & No & 8.8 & Yes \\
\hline Ischaemic heart disease & Patient died & No & 2.8 & No \\
\hline \multicolumn{5}{|l|}{ Glucocorticoid group } \\
\hline \multicolumn{5}{|l|}{ Adverse reactions } \\
\hline Hospitalisation owing to severe reaction after injection & Resolved completely & Probably & 1.0 & No \\
\hline Anaphylactic reaction & Resolved completely & Probably & 0.2 & No \\
\hline Cramp in calves & Resolved partially & Probably & 0.6 & No \\
\hline Sore leg & Resolved completely & Possibly & 0.3 & No \\
\hline Mood swings & Resolved completely & Possibly & 0.1 & No \\
\hline Fever and flu symptoms & NA & Possibly & 0.2 & No \\
\hline \multicolumn{5}{|l|}{ Adverse events } \\
\hline Raised ALT levels & Resolved completely & Possibly & 9.5 & Yes \\
\hline Strangulated hernia & Patient died & Probably not & 13.5 & Yes \\
\hline Rectal bleeding & Not resolved & Probably not & 0.1 & No \\
\hline Intermittent cramp & Not resolved & Probably not & 0.3 & No \\
\hline Rash & Resolved partially & Probably not & 1.6 & No \\
\hline Nausea & Resolved completely & Probably not & 0.5 & No \\
\hline Vomiting & Resolved completely & Probably not & 0.5 & No \\
\hline Pneumonia & Resolved completely & Probably not & 4.0 & No \\
\hline Overdose & Resolved completely & No & 4.2 & Yes \\
\hline Admitted to hospital owing to worsening arthritis & Not resolved & No & 0.7 & Yes \\
\hline Lower respiratory tract infection & Resolved completely & No & 11.1 & Yes \\
\hline Glycosuria & Resolved completely & No & 3.0 & No \\
\hline Chest infection & Resolved completely & No & 2.5 & No \\
\hline Infected sinuses & Resolved completely & No & 3.5 & No \\
\hline Cold & Resolved completely & No & 4.9 & No \\
\hline Urinary tract infection & Not resolved & No & 0.07 & No \\
\hline Intermittent pain and needles right foot & Not resolved & No & 6.0 & Yes \\
\hline Raised ALT levels & Not resolved & No & 5.5 & No \\
\hline
\end{tabular}

${ }^{*}$ Time between baseline visit and occurrence of adverse event; patient used disease-modifying antirheumatic drugs (DMARDs) at time of adverse event (yes or no).

until the actual start of DMARDs for the total population. All statistical analyses were conducted using STATA V9.

\section{RESULTS}

Patients were recruited between October 2002 and October 2006. Of the 572 patients screened, 239 patients did not fulfil the entry criteria, primarily because they had a symptom duration of more than 10 weeks (fig 1). Sixty-five patients did not consent for various reasons. In addition, three patients were excluded from the analyses due to protocol violation. In total, 132 patients were randomised to the placebo group and 133 patients to the glucocorticoid group. At baseline, mean (SD) symptom duration was 8.0 (2.3) weeks in the placebo group and
7.7 (2.6) weeks in the glucocorticoid group (table 1). During this 1-year trial, 20 patients in the placebo group and 21 in the glucocorticoid group were withdrawn from the study (fig 1).

\section{Primary outcome measure}

At 6 months, $76 \%(96 / 127)$ of the patients in the placebo group compared with $61 \%(77 / 126)$ in the glucocorticoid group had either started or been referred to start DMARDs. Overall, patients in the placebo group were more likely to need DMARDs during the first 6 months of the trial than patients in the glucocorticoid group (adjusted $\mathrm{OR}=2.11,95 \% \mathrm{CI} 1.16$ to $3.85, \mathrm{p}=0.015)$. The adjusted $\mathrm{ORs}$ from the sensitivity analyses were $2.07(95 \%$ CI 1.19 to $3.63, p=0.011)$ if all patients with 
missing data were considered not to have needed DMARDs and 2.01 (95\% CI 1.11 to $3.63, p=0.021)$ if all these patients were considered to have needed DMARDs.

\section{Secondary outcome measures}

Both at 6 months and at 1 year, there was no statistically significant difference between the two treatment groups for any of the measures (table 2). Furthermore, there was no difference in the number of patients with no joint damage at baseline who developed erosions between the treatment groups at one year; $9 / 61(14.8 \%)$ in the placebo group and $9 / 71(12.7 \%)$ in the glucocorticoid group ( $p=0.802$, Fisher exact test).

A final diagnosis by the rheumatologist at 1 year was obtained for 222 patients, 111 patients in each group (table 3). In the placebo group $60.4 \%(67 / 111)$ had a clinical diagnosis of RA according to the rheumatologist compared with 48.6\% (54/ 111 ) in the glucocorticoid-treated group (adjusted $\mathrm{OR}=1.58$, $95 \%$ CI 0.85 to $2.93, p=0.145)$. The disease had resolved without the use of DMARDs in $9.9 \%(11 / 111)$ of the patients in the placebo group and $19.8 \%(22 / 111)$ in the glucocorticoidtreated group (adjusted $\mathrm{OR}=0.42,95 \%$ CI 0.18 to 0.99 , $\mathrm{p}=0.048)$.

\section{Additional treatments}

The median survival time until the start of DMARDs was 2.8 months in the placebo group and 8.3 months in the glucocorticoid group ( $p=0.001$; log-rank test) (fig 2 ). In the group of patients who started DMARDs, the average (SD) number of DMARDs taken during the 12 months follow-up period was 1.59 (0.68) in the placebo group and $1.37(0.64)$ in the glucocorticoid group ( $\mathrm{p}=0.067)$, including, respectively, methotrexate $(40.0 \%$ and $52.6 \%$ of all prescribed DMARDs), oral glucocorticoids (13.3\% and $10.3 \%)$, sulfasalazine $(25.9 \%$ and $22.7 \%$ ), hydroxychloroquine $(12.6 \%$ and $11.3 \%)$, leflunomide $(2.2 \%$ and $2.1 \%)$, gold $(3.0 \%$ and $0 \%)$, azathioprine $(1.5 \%$ and $1.0 \%)$, etanercept $(0.7 \%$ and $0 \%)$ and adalimumab $(0.7 \%$ and $0 \%$ ). Data on additional IM and intra-articular glucocorticoid injections were available for 210 patients. Intra-articular injections were given to $16 \%(16 / 103)$ of the glucocorticoid group and $12 \%(13 / 107)$ of the placebo group. Thirty-five per cent $(36 / 103)$ of patients in the glucocorticoid group compared with $45 \%(48 / 107)$ of patients in the placebo group received at least one IM glucocorticoid injection during follow-up. Of those patients with a follow-up duration of more than 1 month, 14 patients did not complete the injection course according to protocol. Five patients received IM glucocorticoid injections, four patients started DMARDs and one patient started DMARDs and received a glucocorticoid injection; all these patients were in the placebo group. One patient refused to receive the last two injections and one patient did not attend the clinic for the last two injections. No further injections were given to two patients because of side effects experienced after the first injection.

\section{Adverse events}

A total of 36 adverse events in 26 patients were reported (table 4). Ten of these were indicated as probably or possibly related to the treatment by the rheumatologist (three in the placebo group and seven in the glucocorticoid group). Three events were defined as serious (unexpected) adverse reactions. In the glucocorticoid group, one patient was hospitalised owing to a severe reaction after the methylprednisolone acetate injection and one patient had an anaphylactic reaction following the methylprednisolone acetate injection. One patient in the placebo group was admitted to the hospital with severe abdominal pain.

\section{DISCUSSION}

The STIVEA trial has shown that a 3-week course of IM methylprednisolone acetate in patients with very early IP is beneficial. Patients who received methylprednisolone acetate injections were less likely to need DMARDs within the first 6 months after inclusion in the study. Although the sample size of the study was based on being able to identify if a 3 week course with IM methylprednisolone acetate injections could prevent one in four patients needing DMARDs by their 6month assessment, we found that the course of methylprednisolone acetate injections could prevent one in five patients from needing DMARDs. This was statistically significant owing to the lower than expected loss to follow-up. We feel that this finding is clinically important. Moreover, the disease had resolved in $19.8 \%$ of the patients in the glucocorticoid group compared with $9.9 \%$ in the placebo group at one year. One of the main objectives in our study was to prevent patients from progressing to RA, whereas most patients in previously published studies already fulfilled the ACR criteria for RA at entry to the study. ${ }^{8-10}$ In another study including only IgM-RF positive arthralgia patients without arthritis, no beneficial effect of IM dexamethasone injections was found in the development of arthritis (see article on page $X X$ ). ${ }^{24} \mathrm{~A}$ difference in results may be explained by the percentage of RF positivity (31-35\%) in our trial, whereas in the arthralgia trial autoantibody positivity was an entry criterion, thereby selecting a category of patients with more severe disease. In contrast to previous studies of IM glucocorticoids in RA, ${ }^{89}$ we did not find statistically significant differences in disease activity and patient health-related outcome measures between the two groups at 6 months. A possible explanation might be that most patients in the placebo group started to use DMARDs relatively early after the study start. Furthermore, five patients in the placebo group received IM glucocorticoid injections within the first 3 weeks of the study owing to worsening of the disease. Therefore, with respect to their arthritis, there was no evidence that patients came to any harm through receiving the three injections of saline.

Two patients $(1.5 \%)$ had a severe reaction to the methylprednisolone acetate injection. IM methylprednisolone acetate injections were otherwise well tolerated, with only a few reported adverse events. Considering the beneficial effects, including postponement of DMARD use, and the low rate of adverse events, a 3-week course of IM methylprednisolone acetate injections could be given to patients who present with soft tissue swelling of two or more joints including a hand joint, and a symptom duration between 4 and 10 weeks. Patients in this trial were only followed up for 1 year and we, therefore, do not know if patients whose disease resolved remained in remission in the future. In general, patients should be advised to return promptly for follow-up if their arthritis recurs or worsens as they may then need DMARD treatment.

One of the main problems of this study was identifying patients with a disease duration of $<11$ weeks. Although the lag time between symptom onset and referral to primary and secondary care has decreased in recent decades, a UK study showed that the median delay before the patient presents in primary care was still 12 weeks in $2006 .{ }^{25}$ To successfully apply the protocol described in this study, it is important that both the general population and general practitioners are aware of the consequences of a delay in assessment. 
We conclude that a 3-week course of IM methylprednisolone acetate injections in patients with very early IP appears to postpone the need for DMARDs and prevent approximately one in 10 patients from progressing into RA within the next 12 months.

Acknowledgements The authors would like to thank all the rheumatologists and research nurses of the participating hospitals and all GPs who referred patients to the rheumatology departments. We also would like to thank all members of the Trial Steering Committee of this study.

Funding This study was funded by the Arthritis Research Campaign UK. Costs for methylprednisolone acetate were covered by Pfizer Ltd.

\section{Competing interests None.}

Ethics approval Approval from the North West Research Ethics Committee, UK.

Current working address MJM-The University of Western Australia, School of Medicine and Pharmacology, 4th Floor G Block, Sir Charles Gairdner Hospital, Hospital Avenue, Nedlands, WA 6009, Australia.

The STIVEA investigators are Cannock Chase Hospital Cannock, Dr Diarmuid Mulherin; Haywood Hospital, Stoke-on-Trent, Dr PT Dawes; Macclesfield District General Hospital, Dr Susan Knight; Kings College Hospital London, Professor David Scott; Royal Cornwall Hospital Truro, Dr Martin Davis; Stepping Hill Hospital Stockport, Dr Jeff Marks; Manchester Royal Infirmary Manchester, Dr lan Bruce; Russells Hall Hospital Dudley, Professor George Kitas; Hope Hospital Salford, Dr Terry O'Neill; Royal Lancaster Infirmary Lancaster, Dr Marwan Bukhari; Norfolk and Norwich University Hospital Norwich, Dr Karl Gaffney; City Hospital Birmingham Birmingham, Dr Karim Raza; Freeman Hospital Newcastle, Dr Lesley Kay; Queen Elizabeth Hospital Gateshead, Dr Clive Kelly and Dr Vadivelu Saravanan; Nevill Hall Hospital Abergavenny, Dr Stuart Linton; Taunton and Somerset Hospital Taunton, Dr Cathy Laversuch; St Helen's Hospital St Helens, Dr Rikki Abernethy; Harold Wood Hospital Romford, Professor Kuntal Chakravarty; Poole General Hospital Poole, Dr Selwyn Richards; St Georges Hospital Tooting, Dr Brian Bourke; The Queen Elizabeth The Queen Mother Hospital Margate, Dr Alison Leak; East Surrey Hospital Redhill, Dr Raad Makadsi; Ysbyty Gwynedd Bangor, Professor Peter Maddison.

Provenance and peer review Not commissioned; externally peer reviewed.

\section{REFERENCES}

1. Symmons DP, Barrett EM, Bankhead CR, et al. The incidence of rheumatoid arthritis in the United Kingdom: results from the Norfolk Arthritis Register. $\mathrm{Br} J$ Rheumatol 1994;33:735-9.

2. Neeck G, Federlin K, Graef V, et al. Adrenal secretion of cortisol in patients with rheumatoid arthritis. J Rheumatol 1990;17:24-9.

3. Crofford LJ, Kalogeras KT, Mastorakos G, et al. Circadian relationships between interleukin (IL)-6 and hypothalamic-pituitary-adrenal axis hormones: failure of IL-6 to cause sustained hypercortisolism in patients with early untreated rheumatoid arthritis. J Clin Endocrinol Metab 1997;82:1279-83.

4. Chikanza IC, Petrou P, Kingsley G, et al. Defective hypothalamic response to immune and inflammatory stimuli in patients with rheumatoid arthritis. Arthritis Rheum 1992;35:1281-8.

5. Boers M, Verhoeven AC, Markusse HM, et al. Randomised comparison of combined step-down prednisolone, methotrexate and sulphasalazine with sulphasalazine alone in early rheumatoid arthritis. Lancet 1997;350:309-18.
6. Kirwan JR. The effect of glucocorticoids on joint destruction in rheumatoid arthritis The Arthritis and Rheumatism Council Low-Dose Glucocorticoid Study Group. N Eng/ J Med 1995;333:142-6.

7. Van Everdingen AA, Jacobs JW, Siewertsz van Reesema DR, et al. Low-dose prednisone therapy for patients with early active rheumatoid arthritis: clinical efficacy, disease-modifying properties, and side effects: a randomized, double-blind, placebocontrolled clinical trial. Ann Intern Med 2002;136:1-12.

8. Corkill MM, Kirkham BW, Chikanza IC, et al. Intramuscular depot methylprednisolone induction of chrysotherapy in rheumatoid arthritis: a 24-week randomized controlled trial. Br J Rheumatol 1990;29:274-9.

9. Choy EH, Kingsley GH, Corkill MM, et al. Intramuscular methylprednisolone is superior to pulse oral methylprednisolone during the induction phase of chrysotherapy. $\mathrm{Br} \mathrm{J}$ Rheumatol 1993:32:734-9.

10. Gough A, Sheeran T, Arthur V, et al. Adverse interaction between intramuscular methylprednisolone and sulphasalazine in patients with early rheumatoid arthritis. A pilot study. Scand J Rheumatol 1994;23:46-8.

11. Choy EH, Kingsley GH, Khoshaba B, et al. A two year randomised controlled trial of intramuscular depot steroids in patients with established rheumatoid arthritis who have shown an incomplete response to disease modifying antirheumatic drugs. Ann Rheum Dis 2005:64:1288-93.

12. Smolen JS, Aletaha D, Machold KP. Therapeutic strategies in early rheumatoid arthritis. Best Pract Res Clin Rheumatol 2005;19:163-77.

13. Quinn MA, Emery P. Window of opportunity in early rheumatoid arthritis: possibility of altering the disease process with early intervention. Clin Exp Rheumatol 2003;21:S154-7.

14. Breedveld FC, Kalden JR. Appropriate and effective management of rheumatoid arthritis. Ann Rheum Dis 2004;63:627-33.

15. Green M, Marzo-Ortega H, McGonagle D, et al. Persistence of mild, early inflammatory arthritis: the importance of disease duration, rheumatoid factor, and the shared epitope. Arthritis Rheum 1999;42:2184-8.

16. Treasure T, MacRae KD. Minimisation: the platinum standard for trials? Randomisation doesn't guarantee similarity of groups; minimisation does. BMJ 1998:317:362-3.

17. Dasgupta B, Gray J, Fernandes L, et al. Treatment of polymyalgia rheumatica with intramuscular injections of depot methylprednisolone. Ann Rheum Dis 1991:50:942-5.

18. Prevoo ML, van 't Hof MA, Kuper HH, et al. Modified disease activity scores that include twenty-eight-joint counts. Development and validation in a prospective longitudinal study of patients with rheumatoid arthritis. Arthritis Rheum 1995;38:44-8.

19. EuroQol - a new facility for the measurement of health-related quality of life. The EuroOol Group. Health Policy 1990;16:199-208.

20. Ware JE Jr, Kosinski MA, Keller SD. SF-36 physical and mental health summary scales: a user's manual. 5th ed. Boston, Massachusetts: Health Assessment Lab, New England Medical Center,1994.

21. Kirwan JR, Reeback JS. Stanford Health Assessment Questionnaire modified to assess disability in British patients with rheumatoid arthritis. Br J Rheumatol 1986;25:206-9.

22. Matthews JNS. Introduction to randomized controlled clinical trials. 2nd ed. London: Arnold, 2009.

23. White IR, Thompson SG. Adjusting for partially missing baseline measurements in randomized trials. Stat Med 2005;24:993-1007.

24. Bos WH, Dijkmans BA, Boers M et al. Effect of dexamethasone on autoantibody levels and arthritis development in arthralgia patients: a randomized trial. Ann Rheum Dis 2010;69:571-4.

25. Kumar K, Daley E, Carruthers DM, et al. Delay in presentation to primary care physicians is the main reason why patients with rheumatoid arthritis are seen late by rheumatologists. Rheumatology (Oxford) 2007;46:1438-40. 